\title{
Die Hodge-Vermutung
}

\author{
Herbert Kurke
}

\section{Einführung}

Bekanntlich ist eine geschlossene, orientierbare Fläche $S$ homöomorph zu einer Sphäre mit $g$ Henkeln, d.h. $S$ lässt sich umkehrbar eindeutig und stetig auf eine Sphäre mit $g$ Henkeln abbilden. Die natürliche Zahl $g$ wird das Geschlecht von $S$ genannt; es ist eine topologische Invariante der Fläche $S$. Dieses Ergebnis lässt sich mit Hilfe der singulären Homologietheorie auch algebraisch beschreiben. Ist $N$ eine $n$-dimensionale, topologische Mannigfaltigkeit, d.h. $N$ ist ein topologischer Hausdorff-Raum, der lokal zum $\mathbb{R}^{n}$ homöomorph ist, so können wir $N$ die singulären Homologiegruppen $H_{k}(N)(k \in \mathbb{Z})$ zuordnen. Diese werden durch gewisse rationale Linearkombinationen $k$-dimensionaler Simplices, den sogenannten geschlossenen $k$-Ketten, erzeugt. Es stellt sich heraus, dass die Isomorphieklassen der $\mathbb{Q}$-Vektorräume $H_{k}(N)$ topologische Invarianten der Mannigfaltigkeit $N$ sind. Im Spezialfall $N=S$ stellen wir $H_{1}(S)$ als $2 g$-dimensional fest, was zur gewünschten algebraischen Interpretation des Geschlechts $g$ führt.

Neben den Homologiegruppen $H_{*}(N)=H_{*}(N, \mathbb{Q})$ mit rationalen Koeffizienten werden dual dazu auch die Kohomologiegruppen $H^{*}(N)=H^{*}(N, \mathbb{Q})$ der Mannigfaltigkeit $N$ betrachtet. Für einen $\mathbb{Q}$-Vektorraum $K$, z.B. $K=\mathbb{R}, \mathbb{C}$, setzen wir weiter $H_{*}(N, K):=$ $H_{*}(N) \otimes K, H^{*}(N, K):=H^{*}(N) \otimes K$. Die Homologie- bzw. Kohomologiegruppen mit Koeffizienten in $K$ besitzen dann folgende Eigenschaften:

(i) Es besteht die Gleichheit $H^{*}(N, K)=\operatorname{Hom}\left(H_{*}(N), K\right)$.

(ii) $H^{*}(N)$ besitzt die Struktur einer graduierten, assoziativen Algebra; das Produkt von $\alpha$ mit $\beta$ ist durch das Cup-Produkt $\alpha \cup \beta$ gegeben. $H_{*}(N, K)$ besitzt die Struktur eines graduierten $H^{*}(N)$-Moduls; die Modulstruktur ist durch das Cap-Produkt $\cap$ gegeben.

(iii) Ist die $n$-dimensionale Mannigfaltigkeit $N$ geschlossen und orientiert, so besitzt $H_{n}(N)$ ein kanonisches erzeugendes Element, die Fundamentalklasse $[N]$; weiter ist $H_{k}(N)=0$ für $k<0$ und $k>n$.

(iv) Poincaré-Dualität: Ist $N$ wiederum geschlossen und orientiert, so besteht eine Isomorphie $H^{k}(N) \cong H_{n-k}(N)$, gegeben durch die Zuordnung $\alpha \mapsto \alpha \cap[N]$.

(v) Ist $M$ eine geschlossene, orientierte, $p$-dimensionale Untermannigfaltigkeit von $N$, so induziert die Einbettung $i: M \rightarrow N$ einen Homomorphismus $i_{*}: H_{p}(M) \rightarrow$ $H_{p}(N)$. Nach (iv) entspricht das Bild der Fundamentalklasse $i_{*}[M] \in H_{p}(N)$ einer 
Kohomologieklasse in $H^{n-p}(N)$, welche wir mit $z(M)$ bezeichnen. Zwischen $M$ und $z(M)$ besteht die Beziehung

$$
(\alpha \cup z(M)) \cap[N]=i^{*} \alpha \cap[M] \quad\left(\alpha \in H^{p}(N)\right) .
$$

Ist $K=\mathbb{R}$ und $N$ eine differenzierbare Mannigfaltigkeit, so lassen sich die Kohomologiegruppen $H^{k}(N, K)$ mit den de Rhamschen Kohomologiegruppen $H_{\mathrm{DR}}^{k}(N, \mathbb{R})$ identifizieren; diese werden durch glatte, geschlossene Differentialformen vom Grad $k$ erzeugt. Legt man auf $N$ noch eine Riemannsche Metrik fest, so besagt der Satz von Hodge, dass für geschlossene, orientierte Mannigfaltigkeiten $N$ jede Kohomologieklasse in $H_{\mathrm{DR}}^{k}(N, \mathbb{R})$ durch genau eine harmonische Differentialform repräsentiert werden kann.

Ist nun $N=X$ eine komplexe, projektiv algebraische Mannigfaltigkeit, d.h. $X$ ist gegeben als Nullstellenmenge homogener Polynome in einem komplexen, projektiven Raum, so geht es bei der Hodge-Vermutung darum, diejenigen Kohomologieklassen $\gamma \in H^{*}(X)$ zu charakterisieren, die Kohomologieklassen von algebraischen Untervarietäten $Y$ sind, d.h. für die $\gamma=z(Y)$ gilt, wobei $Y$ durch polynomiale Gleichungen definiert ist.

Um die Hodge-Vermutung im vierten Abschnitt genau formulieren zu können, besprechen wir im nächsten Abschnitt zunächst den Begriff einer „Hodge-Struktur“ und wenden diesen dann im dritten Abschnitt auf die Kohomologie von sogenannten „KählerMannigfaltigkeiten" an.

\section{Hodge-Strukturen}

Eine reelle Hodge-Struktur vom Gewicht $k$ auf einem endlich dimensionalen reellen Vektorraum $H$ ist durch die folgenden, zueinander äquivalenten Daten gegeben:

(i) Eine Zerlegung von $H_{\mathbb{C}}=H \otimes_{\mathbb{R}} \mathbb{C}$ in eine direkte Summe komplexer Unterräume $H_{\mathbb{C}}=\bigoplus_{p+q=k} H^{p, q}$ mit $H^{q, p}=\overline{H^{p, q}}$ (Hodge-Zerlegung).

(ii) Eine absteigende Filtrierung $\left\{F^{p}\right\}$ von $H_{\mathbb{C}}$ durch komplexe Unterräume $F^{p}$, d.h. $H_{\mathbb{C}} \supseteq \ldots \supseteq F^{p} \supseteq F^{p+1} \supseteq \ldots \supseteq\{0\}$, so dass $F^{p}$ und $\overline{F^{k-p+1}}$ zueinander komplementäre Unterräume sind (Hodge-Filtrierung).

(iii) Eine rationale Darstellung der Gruppe $R=\left\{g=\left(\begin{array}{cc}a & -b \\ b & a\end{array}\right) \in \mathrm{GL}(2, \mathbb{R})\right\}$ auf $H$ vom Gewicht $k$, d.h. ein Homomorphismus $\rho_{H}: R \rightarrow \mathrm{GL}(H)$ mit der Eigenschaft $\rho_{H}\left(\begin{array}{ll}a & 0 \\ 0 & a\end{array}\right)=a^{k} \cdot$ id.

Die Äquivalenz von (i) mit (ii) ergibt sich aus $F^{p}=\bigoplus_{r \geq p} H^{r, s}$ bzw. $H^{p, q}=F^{p} \cap \overline{F^{q}}$. Die Äquivalenz von (i) mit (iii) ergibt sich aus der Tatsache, dass $H^{p, q}$ Eigenraum zum Charakter $z^{p} \bar{z}^{q}: R \rightarrow \mathbb{C}^{*}$ mit $z(g)=a+i b, \bar{z}(g)=a-i b$ ist.

Eine Hodge-Struktur vom Gewicht $k$ ist eine reelle Hodge-Struktur $H$, in der ausserdem ein Gitter $H_{\mathbb{Z}} \subset H$, d.h. eine Untergruppe, die von einer Basis von $H$ erzeugt wird, ausgezeichnet ist. Morphismen von Hodge-Strukturen $H, H^{\prime}$ sind $\mathbb{R}$-lineare Abbildungen $\varphi: H \rightarrow H^{\prime}$ mit $\varphi\left(H_{\mathbb{Z}}\right) \subseteq H_{\mathbb{Z}}^{\prime}$, die nach $\mathbb{C}$-linearer Fortsetzung die Hodge-Zerlegung erhalten, also $\varphi\left(H^{p, q}\right) \subseteq H^{\prime} p, q$ bzw. $\varphi \circ \rho_{H^{\prime}}=\rho_{H} \circ \varphi$ erfüllen. 


\section{Kähler-Mannigfaltigkeiten und projektive algebraische Mannigfaltigkeiten}

Es sei $X$ eine komplexe Mannigfaltigkeit. Die komplexe Struktur induziert rationale Darstellungen vom Gewicht 1 der Gruppe $R$ (Abschnitt 2, (iii)) auf den Tangentialund Kotangentialräumen von $X$, als reelle glatte Mannigfaltigkeit aufgefasst, so dass $C=\varrho\left(\begin{array}{cc}0 & -1 \\ 1 & 0\end{array}\right)$ der Multiplikation mit $i$ entspricht. Daher wird eine Zerlegung des Raumes der glatten Differentialformen induziert, d.h.

$$
A^{k}(X, \mathbb{C})=\bigoplus_{p+q=k} A^{p, q}(X)
$$

Bezeichnen $z=\left(z_{1}, \ldots, z_{n}\right)$ lokale holomorphe Koordinaten auf $X$, so ist eine $(p, q)$ Form $\eta$ lokal gegeben durch

$$
\eta=\sum_{\substack{1 \leq i_{1}<\ldots<i_{p} \leq n \\ 1 \leq j_{1}<\ldots<j_{q} \leq n}} f_{i_{1}, \ldots, i_{p} ; j_{1}, \ldots, j_{q}}(z, \bar{z}) d z_{i_{1}} \wedge \ldots \wedge d z_{i_{p}} \wedge d \bar{z}_{j_{1}} \wedge \ldots \wedge d \bar{z}_{j_{q}}
$$

wobei $f_{i_{1}, \ldots, i_{p} ; j_{1}, \ldots, j_{q}}(z, \bar{z})$ glatte, komplexwertige Funktionen sind.

Indem wir $X$ als $2 n$-dimensionale, differenzierbare Mannigfaltigkeit auffassen, können wir $X$ mit einer Riemannschen Metrik $g$ versehen. Eine Metrik heisst Kählersch, wenn die Bilinearform $\omega(v, w)=g(C v, w)$ alternierend und geschlossen ist, d.h. die Gleichung $d \omega=0$ erfüllt; hierbei sind $v, w$ Tangentialvektoren. Wir nennen $\omega$ eine Kähler-Form und das Paar $(X, \omega)$ eine Kähler-Mannigfaltigkeit, die Metrik ist dann $g(v, w)=\omega(v, C w)$. Ein wichtiges Beispiel ist der komplexe projektive Raum $\mathbb{P}^{n}(\mathbb{C})$ versehen mit der FubiniStudy-Metrik $\|\cdot\|$; in diesem Fall gilt

$$
\omega=\frac{i}{2 \pi} \cdot \partial \bar{\partial} \log \left(\frac{\|z\|^{2}}{\left|z_{0}\right|^{2}}\right)
$$

für $z=\left(z_{0}: \ldots: z_{n}\right) \in \mathbb{P}^{n}(\mathbb{C})$ mit $z_{0} \neq 0$. Die Fubini-Study-Metrik induziert auf jeder komplexen Untermannigfaltigkeit $X \subseteq \mathbb{P}^{n}(\mathbb{C})$ eine Kähler-Metrik, so dass all diese Kähler-Mannigfaltigkeiten sind. Nach einem Satz von W.L. Chow sind die abgeschlossenen, analytischen Untervarietäten von $\mathbb{P}^{n}(\mathbb{C})$ genau die glatten, algebraischen Untervarietäten von $\mathbb{P}^{n}(\mathbb{C})$ (siehe [6]).

Jede Kähler-Metrik einer kompakten Kähler-Mannigfaltigkeit $(X, \omega)$ definiert nicht verschwindende Kohomologieklassen

$$
\left[\omega^{k}\right] \in H_{\mathrm{DR}}^{2 k}(X, \mathbb{R}) \quad(k=1, \ldots, \operatorname{dim}(X)) .
$$

Wenn $[\omega] \in H^{2}(X, \mathbb{Q})$ ist, so heisst $(X, \omega)$ eine Hodge-Mannigfaltigkeit. Dies ist z.B. für projektiv algebraische Mannigfaltigkeiten der Fall; daher taucht für den projektiven Raum $\mathbb{P}^{n}(\mathbb{C})$ in der Formel für $\omega$ der Normierungsfaktor $1 / \pi$ auf. Nach dem Einbettungssatz von Kodaira gilt nun auch die Umkehrung: Wenn für eine geschlossene KählerMannigfaltigkeit $(X, \omega)$ die Klasse $[\omega]$ rational ist, d.h. $[\omega] \in H^{2}(X, \mathbb{Q})$ gilt, so besitzt $X$ 
eine projektive Einbettung, so dass ein Vielfaches $k \cdot[\omega]$ von $[\omega]$ die Fubini-Study-Klasse enthält.

Das Besondere im Falle von kompakten Kähler-Mannigfaltigkeiten $(X, \omega)$ ist das folgende: Definiert man den Operator $L$ durch $L(\alpha):=\omega \wedge \alpha$ und bezeichnet mit $\Lambda$ den zu $L$ (punktweise) adjungierten Operator, so zeigt sich, dass im Kählerschen Fall der LaplaceOperator $\Delta$ (ein natürlicher Differentialoperator 2. Ordnung) sowohl mit den Operatoren $L$ und $\Lambda$ als auch mit der Operation der Gruppe $R$ aus Abschnitt 2 vertauscht. Als Folge daraus erhält man für kompakte, $n$-dimensionale Kähler-Mannigfaltigkeiten:

(i) $H^{k}(X, \mathbb{R})$ besitzt eine kanonische Hodge-Strukur vom Gewicht $k$, d.h. $H^{k}(X, \mathbb{C})=$ $\bigoplus_{p+q=k} H^{p, q}(X)$, wobei die Räume $H^{p, q}(X)$ durch gewisse $(p, q)$-Formen erzeugt werden.

(ii) Die Kähler-Form $\omega$ induziert zueinander adjungierte Operatoren $L$ bzw. $\Lambda$ auf $H^{*}(X, \mathbb{R})$ vom Grad 2 bzw. -2 (genauer: $L \in \operatorname{Hom}\left(H^{k}(X, \mathbb{R}), H^{k+2}(X, \mathbb{R})\right)^{(1,1)}$ bzw. $\left.\Lambda \in \operatorname{Hom}\left(H^{k}(X, \mathbb{R}), H^{k-2}(X, \mathbb{R})\right)^{(-1,-1)}\right)$.

(iii) Durch die Zuordnung

$$
\left(\begin{array}{cc}
-a & b \\
c & a
\end{array}\right) \mapsto a \Pi+b \Lambda+c L \quad\left(\text { mit } \Pi=(k-n) \cdot \operatorname{id} \text { auf } H^{k}(X, \mathbb{R})\right)
$$

wird $H^{*}(X, \mathbb{R})$ zu einer Darstellung der Lie-Algebra $\mathfrak{I}(2, \mathbb{R})$.

(iv) Die Abbildung $H^{k}(X, \mathbb{R}) \stackrel{L}{\longrightarrow} H^{k+2}(X, \mathbb{R})$ ist injektiv für $k \leq n-1$ und surjektiv für $k \geq n-1$; die Abbildung $H^{p, q}(X) \stackrel{L}{\longrightarrow} H^{p+1, q+1}(X)$ ist injektiv für $p+q \leq n-1$ und surjektiv für $p+q \geq n-1$.

(v) Harter Lefschetz-Satz: Die Abbildung $H^{n-k}(X, \mathbb{R}) \stackrel{L^{k}}{\longrightarrow} H^{n+k}(X, \mathbb{R})$ ist ein Isomorphismus.

\section{Die Hodge-Vermutung}

\subsection{Zyklenabbildung, Niveau-Filtrierung}

Es sei $X$ wiederum eine $n$-dimensionale, komplexe Mannigfaltigkeit und $Y \subseteq X$ eine analytische Untervarietät der Kodimension $\operatorname{kodim}(Y, X)=n-\operatorname{dim}(Y)=p$. Die Untervarietät $Y$ definiert nach dem im ersten Abschnitt Gesagten eine Kohomologieklasse $z(Y) \in H^{2 p}(X)$. Weiter besteht eine exakte Sequenz

$$
\ldots \longrightarrow H^{k}(Y) \longrightarrow H^{k+2 p}(X) \longrightarrow H^{k+2 p}(X \backslash Y) \longrightarrow \ldots
$$

Die Niveau-Filtrierung $N^{p} H^{*}(X)$ von $H^{*}(X)$ wird dann durch

$$
N^{p} H^{*}(X)=\sum_{\substack{Y \subseteq X \\ \operatorname{kodim}(\bar{Y}, X) \geq p}} \operatorname{Ker}\left(H^{*}(X) \longrightarrow H^{*}(X \backslash Y)\right)
$$

definiert. Sie ist über $\mathbb{Q}$, ja sogar über $\mathbb{Z}$, definiert; daher gilt $N^{p} H^{*}(X, K)=N^{p} H^{*}(X) \otimes$ $K$ für $K=\mathbb{R}, \mathbb{C}$. Es lässt sich nun zeigen, dass die Niveau-Filtrierung feiner als die Hodge-Filtrierung ist, d.h. es besteht die Inklusion

$$
N^{p} H^{*}(X, \mathbb{C}) \subseteq F^{p} H^{*}(X)=\bigoplus_{r \geq p} H^{r, s}(X) .
$$


Weiter stellt man $N^{p} H^{m}(X, \mathbb{Q})=0$ für $m<2 p$ fest; damit erhalten wir für $k \geq 0$

$$
N^{p} H^{2 p+k}(X, \mathbb{C}) \subseteq H^{p, p+k}(X) \oplus H^{p+1, p-1+k}(X) \oplus \cdots \oplus H^{p+k, p}(X) .
$$

Speziell erkennen wir $z(Y)$ als ein Element von $H^{2 p}(X, \mathbb{Q}) \cap H^{p, p}(X)$.

\subsection{Hodge-Vermutung}

Ist $X$ eine komplexe Mannigfaltigkeit und $Y \subseteq X$ eine analytische Untervarietät, so haben wir soeben festgestellt, dass $z(Y) \in H^{2 p}(X, \mathbb{Q}) \cap H^{p, p}(X)$ gilt. Die populäre Form der Hodge-Vermutung ist nun die Umkehrung diese Sachverhalts (siehe [4]):

Hodge-Vermutung: Der Durchschnitt $H^{2 p}(X, \mathbb{Q}) \cap H^{p, p}(X)$ wird durch die Kohomologieklassen $z(Y)$, die zu algebraischen Untervarietäten $Y \subseteq X$ gehören, erzeugt.

Die von Hodge 1950 formulierte Vermutung wurde 1969 durch A. Grothendieck präzisiert (siehe [3]):

Allgemeine Hodge-Vermutung: Für $m \geq 2 p$ ist jede über $\mathbb{Q}$ definierte Unter-HodgeStruktur $V \subseteq H^{m}(X, \mathbb{Q})$ von $H^{m}(X, \mathbb{R})$ mit $V^{j, k}=0$ für $|j-k|>m-2 p$ in $N^{p} H^{m}(X, \mathbb{Q})$ enthalten.

Für $m=2 p$ fällt die allgemeine Hodge-Vermutung mit der populären Form zusammen. Die ursprüngliche Vermutung, dass $N^{p} H^{m}(X, \mathbb{Q})$ selbst eine Unter-Hodge-Struktur von $H^{m}(X)$ ist, hat Grothendieck anhand eines Beispiels, Produkt von 3 geeignet gewählten elliptischen Kurven, widerlegt. Hodge selbst hatte seine Vermutung für ganzzahlige Kohomologieklassen formuliert; dies war zu optimistisch, wie M.F. Atiyah und F. Hirzebruch in [1] gezeigt haben, so dass die Vermutung auf rationale Kohomologieklassen bezogen wurde.

\subsection{Der Fall $m=2$}

Ist $X$ eine kompakte Kähler-Mannigfaltigkeit und $\mathscr{L}$ ein komplexes Geradenbündel auf $X$, so findet man dazu einen Zusammenhang $\nabla$, so dass dessen Krümmung $\nabla \circ \nabla$ eine Form vom Typ $(1,1)$ ist. Zerlegt man $\nabla$ in Formen vom Typ $(1,0)$ und $(0,1)$, d.h.

$$
\nabla=\nabla^{1,0}+\nabla^{0,1}
$$

so folgt $\nabla^{0,1} \circ \nabla^{0,1}=0$. Dies ist aber gerade die Integrabilitätsbedingung für die Existenz lokaler, nicht verschwindender Schnitte $s$ von $\mathscr{L}$ mit $\nabla^{0,1} s=0$, welche zu einer holomorphen Struktur auf $\mathscr{L}$ führt. Mit Hilfe solcher Schnitte können wir nun die erste Chern-Form und somit die erste Chern-Klasse $c_{1}(\mathscr{L})$ von $\mathscr{L}$ definieren; sie ist eine Kohomologieklasse vom Typ $(1,1)$. Indem wir die Gruppe der Isomorphieklassen holomorpher Geradenbündel auf $X$ mit $\operatorname{Pic}(X)$ bezeichnen, erhalten wir das folgende Ergebnis:

Auf kompakten Kähler-Mannigfaltigkeiten $X$ ist das $\operatorname{Bild}$ von $\operatorname{Pic}(X)$ unter $c_{1}$ in $H_{\mathrm{DR}}^{2}(X, \mathbb{R})$ gleich dem Durchschnitt von $H^{1,1}(X)$ mit dem Bild von $H^{2}(X, \mathbb{Z})$.

Auf projektiv algebraischen Mannigfaltigkeiten besitzt nach [6] jedes holomorphe Geradenbündel $\mathscr{L}$ einen meromorphen Schnitt $s \neq 0$. Diesem Schnitt lässt sich ein Divisor $\operatorname{div}(s)=\sum_{\alpha} n_{\alpha} V_{\alpha}$ zuordnen; diese Schreibweise bedeutet, dass der Schnitt $s$ entlang $V_{\alpha}$ 
eine $n_{\alpha}$-fache Nullstelle bzw. Polstelle hat, wenn $n_{\alpha}>0$ bzw. $n_{\alpha}<0$ ist. Zwischen der ersten Chern-Klasse von $\mathscr{L}$ und dem Divisor $\operatorname{div}(s)$ besteht nun der Zusammenhang

$$
c_{1}(\mathscr{L})=\sum_{\alpha} n_{\alpha} z\left(V_{\alpha}\right)
$$

was gerade die Bestätigung der Hodge-Vermutung für Kozyklen der Kodimension 2 bedeutet.

Für $X=\mathbb{P}^{n}(\mathbb{C})$ ist die Kähler-Form $\omega$ der Fubini-Study-Metrik die erste Chern-Form des Hopf-Bündels. Damit sind die Operatoren $L$ und $\Lambda$ auf projektiven algebraischen Varietäten über $\mathbb{Q}$ definiert, also gilt der harte Lefschetz-Satz bereits für $H^{*}(X, \mathbb{Q})$. Der Operator $L$ entspricht dabei dem Cup-Produkt mit der Zykel-Klasse $z(H)$ eines Hyperebenenschnittes $H$. Aufgrund des harten Lefschetz-Satzes gilt dann die HodgeVermutung also auch für Kozyklen der Kodimension 2. Darüber hinaus sind bis heute aber nur Spezialfälle bekannt, in denen die Hodge-Vermutung richtig ist.

\section{Literatur}

[1] Atiyah, M.F.; Hirzebruch, F.: Analytic cycles on complex manifolds. Topology 1 (1962), 25-45.

[2] Deligne, P.: The Hodge conjecture. http://www.claymath.org/prizeproblems/hodge.htm

[3] Grothendieck, A.: Hodge's general conjecture is false for trivial reasons. Topology 8 (1969), 299-303.

[4] Hodge, W.V.D.: The topological invariants of algebraic varieties. Proc. ICM (1950), 181-192.

[5] Kodaira, K.; Spencer, D.C.: Divisor classes on algebraic varieties. Proc. Nat. Acad. Sci. 39 (1953), 872-877.

[6] Serre, J.P.: Géométrie algébrique et géométrie analytique. Ann. Inst. Fourier, Grenoble 6 (1956), 1-42.

[7] Weil, A.: Introduction à l'étude des variétés Kaehleriennes. Publ. Univ. Nancago VI (1958).

Herbert Kurke

Institut für Mathematik

Humboldt-Universität zu Berlin

D-10099 Berlin

e-mail: kurke@mathematik.hu-berlin.de 